\title{
KEYNOTE-021 cohorts $D$ and $H$ suggest modest benefit in combining ipilimumab with pembrolizumab in second-line or later advanced non-small cell lung cancer treatment
}

\author{
Amy Lauren Cummings ${ }^{1}$, Kate M. Santoso ${ }^{2}$, Jonathan W. Goldman ${ }^{1}$ \\ ${ }^{1}$ David Geffen School of Medicine, University of California, Los Angeles, CA, USA; ${ }^{2}$ University of California, Los Angeles, CA, USA \\ Correspondence to: Jonathan W. Goldman, MD. Associate Professor, Department of Medicine, Division of Hematology/Oncology, David Geffen \\ School of Medicine at the University of California, 2020 Santa Monica Blvd, Suite 600, Santa Monica, Los Angeles, CA 90404, USA. Email: \\ jwgoldman@mednet.ucla.edu. \\ Comment on: Gubens MA, Sequist LV, Stevenson JP, et al. Pembrolizumab in combination with ipilimumab as second-line or later therapy for \\ advanced non-small-cell lung cancer: KEYNOTE-021 cohorts D and H. Lung Cancer 2019;130:59-66.
}

Submitted Aug 02, 2019. Accepted for publication Aug 16, 2019.

doi: $10.21037 /$ tlcr.2019.08.11

View this article at: http://dx.doi.org/10.21037/tlcr.2019.08.11

With 15 new non-small cell lung cancer (NSCLC) United States Food \& Drug Association (FDA) approvals in the past 5 years, the alignment of academia, pharmaceutical companies, and government regulatory agencies to develop better cancer therapies and quickly make them available to patients has led to a rapidly evolving standard-of-care (1). While undoubtedly beneficial to NSCLC patients, one ramification is that the environment in which a clinical trial is designed may not be same one in which its results are reported. Such is the case with KEYNOTE-021 Cohorts D and $\mathrm{H}(\mathrm{KN}-021 \mathrm{D} / \mathrm{H})(2)$.

As part of a multi-center, multi-armed phase $1 / 2$ study, $\mathrm{KN}-021 \mathrm{D} / \mathrm{H}$ explored the addition of ipilimumab, a cytotoxic T-lymphocyte associated protein-4 (CTLA-4) antibody, to pembrolizumab, a programmed death 1 (PD-1) antibody, as second-line or later treatment in advanced NSCLC. Exploiting complementary pathways in immune regulation, ipilimumab's CTLA-4 blockade enhances T-cell/antigen-presenting cell (APC) co-stimulation by preventing competition for APC's B7 binding site in lymph nodes, leading to activation and proliferation of multiple T-cell clones, while pembrolizumab's PD-1 blockade prevents $\mathrm{T}$-cell deactivation following recognition of tumor neoantigen in peripheral tissues, restoring the activity of quiescent antitumor T-cells (3). Initially piloted as first-line therapy in advanced melanoma in CheckMate 067, nivolumab plus ipilimumab was compared to nivolumab alone and ipilimumab alone and featured robust improvements in response and survival at the price of increased treatment-related adverse events (AEs) (4). Overall response rate (ORR) was $58 \%$ vs. $45 \%$ vs. $19 \%$ respectively, median progression-free survival (PFS) 11.5 vs. 6.9 vs. 2.9 months, and median overall survival (OS) not met with $71 \%$ alive at 48 months vs. 36.9 vs. 19.9 months when updated at the European Society for Medical Oncology in $2018(4,5)$. Nevertheless, grade 3-4 treatment-related events occurred in $55 \%$ of the nivolumab plus ipilimumab group leading to discontinuation in $36.4 \%$, more than the $16.3 \%$ with $7.7 \%$ discontinuing in the nivolumab group and $27.3 \%$ with $14.8 \%$ discontinuing in the ipilimumab group combined. Yet given the significant survival benefit and NSCLC's similar mutation profile and immunotherapy response pattern, it was assumed NSCLC was a good candidate for dual pathway inhibition (6).

$\mathrm{KN}-021$ opened in 2014 and notably enrolled patients not previously treated with immunotherapy. Participants in the dose selection Cohort D received pembrolizumab $10 \mathrm{mg} / \mathrm{kg}$ with ipilimumab $1 \mathrm{mg} / \mathrm{kg}(\mathrm{n}=3)$, pembrolizumab $10 \mathrm{mg} / \mathrm{kg}$ with ipilimumab $3 \mathrm{mg} / \mathrm{kg}$ ( $\mathrm{n}=3$ ), or pembrolizumab $2 \mathrm{mg} / \mathrm{kg}$ with ipilimumab $1 \mathrm{mg} / \mathrm{kg}(\mathrm{n}=12)$ every 3 weeks for 4 cycles, followed by pembrolizumab monotherapy every 3 weeks for up to 2 years. While no significant dose limiting toxicities occurred at higher dose levels, concerns over treatmentrelated deaths in the similar CheckMate 012 study led to 
the selection of pembrolizumab $2 \mathrm{mg} / \mathrm{kg}$ with ipilimumab $1 \mathrm{mg} / \mathrm{kg}$ for expansion Cohort $\mathrm{H}(\mathrm{n}=33)$.

Forty-four patients were included in the efficacy analysis with an ORR primary endpoint compared to a historical control of $20 \%$ from the pembrolizumab phase $1 \mathrm{~b}$ study; 45 patients received at least one recommended dose and were eligible for safety analyses (including one participant that was found to have melanoma). The primary endpoint of the study was not met by the ORR of $30 \%(13 / 44, \mathrm{P}=0.0858)$. ORR also did not clearly correlate with PD-ligand 1 (PD-L1) tumor proportion score (TPS) by the $22 \mathrm{C} 3$ assay-those with a TPS $<1 \%$ had an ORR of $25 \%(5 / 20)$; for TPS $1-49 \%$, ORR was $39 \%$ (7/18); and for TPS $\geq 50 \%$, ORR was $17 \%(1 / 6)$. Median PFS was 4.1 months $(95 \%$ CI: 1.4-5.8); median OS was 10.9 months (95\% CI: 6.1-23.7). Grade 3-5 treatment-related AEs occurred in 13/45 (29\%) with one death attributed to pancreatitis after 1 year of therapy. Treatment-related AEs also led to treatment discontinuation in 9/45 (20\%) of patients. With these data, the authors concluded that there was evidence of antitumor activity with pembrolizumab and ipilimumab for advanced NSCLC but with meaningful and concerning toxicity.

$\mathrm{KN}-021 \mathrm{D} / \mathrm{H}$ authors point to unfavorable characteristics of the study population as a likely reason for primary endpoint failure, highlighting the inclusion of sensitizing mutations, negatively skewed PD-L1 TPS in the expansion cohort, and the heavily pretreated nature of the cohort. Epidermal growth factor receptor $(E G F R)$ mutations and anaplastic lymphoma kinase $(A L K)$ rearrangements are known predictors of poor immunotherapy response (7). Present in $27 \%$ (12/44) of study patients, only one of these participants had an objective response. Additionally, almost half of the patients treated at the recommended dose had a PD-L1 TPS $<1 \%$, a biomarker associated with limited response to immunotherapy, and only $6 / 44$ (13.6\%) had a PD-L1 TPS $\geq 50 \%$, which is typically expected in a third of patients and associated with increased ORR (8). Although the PD-L1 high and $<1 \%$ groups performed similarly with ORRs of $20 \%$ and $17 \%$ respectively, limited numbers in the PD-L1 high group prevent any definitive conclusions. Finally, three out of every 4 patients $(73 \%, 32 / 44)$ had received $\geq 2$ lines of prior therapy and $39 \%(17 / 44)$ had $\geq 3$ lines of therapy, corresponding to an ORR of $26 \%(8 / 31)$. While less than the ORR of $38 \%(5 / 13)$ in those with $<2$ lines of therapy, it is slightly more favorable when compared to single-agent pembrolizumab's ORR of $18 \%$ in those with 2 or more lines of therapy in KEYNOTE-010 (9). Nevertheless, the odds were not in KN-021 D/H's favor.
Dose-selection deserves additional attention as it is a significant concern with combination CTLA-4 blockade and represents a deviation from the dose-escalation plan of the protocol. While there were no dose-limiting toxicities in Cohort D at the pembrolizumab $10 \mathrm{mg} / \mathrm{kg}$ and ipilimumab $3 \mathrm{mg} / \mathrm{kg}$ level, the authors selected the pembrolizumab $2 \mathrm{mg} / \mathrm{kg}$ with ipilimumab $1 \mathrm{mg} / \mathrm{kg}$ for expansion Cohort $\mathrm{H}$, but also reported that in those treated with pembrolizumab $10 \mathrm{mg} / \mathrm{kg}$, the ORR was $50 \%(3 / 6)$. While suggestive, the higher ORR is likely spurious and the result of small numbers as pembrolizumab dose is an unlikely contributor to efficacy, especially given pharmacokinetic equivalence studies (10). Further, the level of toxicity seen in Cohort $\mathrm{H}$ at the lowest dose level suggests higher doses would not have been better tolerated, reinforcing the appropriateness of the expansion cohort dose. The authors reason that the every 3 week dosing of ipilimumab was ultimately what was problematic in the trial, citing the CheckMate 012 experience that supported significant dose-limiting toxicities with every 3-week dosing of ipilimumab while 6- or 12week dosing was better tolerated (11).

Irrespective of these nuances, the biggest issue with $\mathrm{KN}$ $021 \mathrm{D} / \mathrm{H}$ is that shortly before its publication, the results of CheckMate 012, MYSTIC, and several other studies failed to find robust clinical activity of CTLA-4 antibodies in combination with other treatments for NSCLC (Table 1) (11-13). In this context, $\mathrm{KN}-021 \mathrm{D} / \mathrm{H}$ 's trial offers more of the same-two out of every three patients discontinue treatment due to toxicity or progression, and there are no suitable biomarkers for robust response. In sum, these results suggest that the addition of CTLA-4 inhibition to PD-1 blockade is not as efficacious in NSCLC as it is in melanoma, which is likely associated with subtle differences in the immunogenicity and mutagenesis of these histologies. Unfortunately, with KN-021 D/H's exclusion of patients with prior immunotherapy use, it also does not address any current clinical need as immunotherapy has moved from second line to first line with FDA-approval of the KEYNOTE-189/407 and IMpower150 regimens (14-16). For patients that have progressed on these chemoimmunotherapy combinations, conventional secondline options remain docetaxel with or without ramucirumab or gemcitabine.

So is combination CTLA-4 and PD-(L) 1 inhibition done for in advanced NSCLC? Not quite yet. Enhanced patient selection and more judicious dosing schedules have improved outcomes. In CheckMate 227, selecting for tumor mutation burden $\geq 10$ mutations/megabase [high 
Table 1 Advanced NSCLC clinical trials involving combination CTLA-4/PD-1 inhibition

\begin{tabular}{|c|c|c|c|c|c|c|}
\hline Lead author, year & Study type & Line & Intervention & Biomarker & Outcome $^{*}$ & Grade 3+ TRAE \\
\hline $\begin{array}{l}\text { Hellman 2018, } \\
\text { CheckMate } 227\end{array}$ & $\begin{array}{l}\text { Randomized, } \\
\text { phase } 3\end{array}$ & $1 \mathrm{st}$ & $\begin{array}{c}\text { Nivolumab + ipilimumab vs. } \\
\text { chemotherapy }\end{array}$ & $\begin{array}{l}\text { TMB, PD-L1 } \\
(28-8 \mathrm{IHC})\end{array}$ & $\begin{array}{c}\text { PFS } 7.2 \text { vs. } 5.5 \text { m; 1-year } \\
\text { PFS } 42.5 \% \text { vs. } 13.2 \%\end{array}$ & $31.2 \%$ vs. $36.1 \%$ \\
\hline $\begin{array}{l}\text { Hellman 2017, } \\
\text { CheckMate } 012\end{array}$ & $\begin{array}{l}\text { Open-label, } \\
\text { phase 1b }\end{array}$ & $1 \mathrm{st}$ & $\begin{array}{l}\text { Nivolumab + ipilimumab } \\
\quad(q 6 w \text { vs. } q 12 w)\end{array}$ & $\begin{array}{c}\text { PD-L1 } \\
(28-8 \mathrm{IHC})\end{array}$ & $\begin{array}{c}\text { ORR 38-47\% } \\
\text { (PD-L1 } \geq 1 \% \text { : ORR 57\%) }\end{array}$ & $\begin{array}{c}37 \%, 33 \% \\
\text { (grade } 5=3 \text { ) }\end{array}$ \\
\hline Antonia 2016 & $\begin{array}{l}\text { Open-label, } \\
\text { phase } 1 b\end{array}$ & $2+$ & $\begin{array}{l}\text { Durvalumab }(3 / 10 / 15 / 20 \mathrm{mg} / \mathrm{kg})+ \\
\text { tremelimumab }(1 / 3 / 10 \mathrm{mg} / \mathrm{kg})\end{array}$ & $\begin{array}{c}\text { PD-L1 } \\
\text { (SP263 IHC) }\end{array}$ & $\begin{array}{c}\text { ORR } 23 \% \\
\text { (no PD-L1 association) }\end{array}$ & $\begin{array}{l}30 \%, 59 \%, 78 \% \\
\quad(\text { grade } 5=3)\end{array}$ \\
\hline
\end{tabular}

*, all results significant unless otherwise noted. IHC, immunohistochemistry; PFS, progression-free survival; ORR, overall response rate; OS, overall survival; PD-L1, programmed death ligand-1; TMB, tumor mutation burden (high $\geq 10$ mutations/ megabase); TRAE, treatment-related adverse events; q, every; w, weeks; m, month.

tumor mutation burden (TMB)] led to an ORR of $45.3 \%$ and median PFS of 7.2 months with first-line nivolumab and ipilimumab compared to $26.9 \%$ and 5.5 months with standard chemotherapy (17). These numbers have since been supported by CheckMate 568, which also showed an ORR of $47-48 \%$ and median PFS of 7.2 months in those with high TMB (18). Yet these studies are hampered by their comparator arms of chemotherapy, and given the robust KEYNOTE-189 survival data, it is hard to imagine a CTLA4 and $\mathrm{PD}-(\mathrm{L}) 1$ combination besting this regimen in a headto-head comparison. Further, FDA approval of nivolumab with ipilimumab has stalled given updated data showing similar survival outcomes in TMB low and high patients, calling the utility of this biomarker into question (19). Whether these issues will be sorted out by improved biomarker selection in the second line is yet to be seen. In the meantime, we will await the results of KEYNOTE-598, which will evaluate first-line pembrolizumab $200 \mathrm{mg}$ every 3 weeks plus ipilimumab $1 \mathrm{mg} / \mathrm{kg}$ once every 6 weeks in a randomized, placebo-controlled phase 3 study of advanced NSCLC patients with PD-L1 TPS $\geq 50 \%$.

\section{Acknowledgments}

None.

\section{Footnote}

Conflicts of Interest: JW Goldman: research grants from Merck, Bristol-Myers Squibb, Genentech and Astra Zeneca; advisor for Genentech and Astra Zeneva; and speakers board for Merck. The other authors have no conflicts of interest to declare.

Ethical Statement: The authors are accountable for all aspects of the work in ensuring that questions related to the accuracy or integrity of any part of the work are appropriately investigated and resolved.

\section{References}

1. Hilal T, Sonbol MB, Prasad V. Analysis of Control Arm Quality in Randomized Clinical Trials Leading to Anticancer Drug Approval by the US Food and Drug Administration. JAMA Oncol 2019;5:887-92.

2. Gubens MA, Sequist LV, Stevenson JP, et al. Pembrolizumab in combination with ipilimumab as second-line or later therapy for advanced non-small-cell lung cancer: KEYNOTE-021 cohorts D and H. Lung Cancer 2019;130:59-66.

3. Buchbinder EI, Desai A. CTLA-4 and PD-1 Pathways: Similarities, Differences, and Implications of Their Inhibition. Am J Clin Oncol 2016;39:98-106.

4. Larkin J, Chiarion-Sileni V, Gonzalez R, et al. Combined Nivolumab and Ipilimumab or Monotherapy in Untreated Melanoma. N Engl J Med 2015;373:23-34.

5. Hodi FS, Chiarion-Sileni V, Gonzalez R, et al. Overall survival at 4 years of follow-up in a phase 3 trial of nivolumab plus ipilimumab combination therapy in advanced melanoma (CheckMate 067). ESMO 2018 
Congress, 22 October 2018. Available online: https:// oncologypro.esmo.org/Meeting-Resources/ESMO2018-Congress/Overall-survival-at-4-years-of-followup-in-a-phase-3-trial-of-nivolumab-plus-ipilimumabcombination-therapy-in-advanced-melanomaCheckMate-067. Accessed 25 July 2019.

6. Heist R. PD-(L)1 inhibitors and CTLA-4 inhibitors: rationale for combinations and recent data in non-small cell lung cancer. Am J Hematol Oncol 2015;11:21-5.

7. Gainor JF, Shaw AT, Sequist LV, et al. EGFR Mutations and ALK Rearrangements Are Associated with Low Response Rates to PD-1 Pathway Blockade in Non-Small Cell Lung Cancer: A Retrospective Analysis. Clin Cancer Res 2016;22:4585-93.

8. Garon EB, Rizvi NA, Hui R, et al. Pembrolizumab for the treatment of non-small-cell lung cancer. N Engl J Med 2015;372:2018-28.

9. Herbst RS, Baas P, Kim DW, et al. Pembrolizumab versus docetaxel for previously treated, PD-L1-positive, advanced non-small-cell lung cancer (KEYNOTE-010): a randomised controlled trial. Lancet 2016;387:1540-50.

10. Freshwater T, Kondic A, Ahamadi M, et al. Evaluation of dosing strategy for pembrolizumab for oncology indications. J Immunother Cancer 2017;5:43.

11. Hellmann MD, Rizvi NA, Goldman JW, et al. Nivolumab plus ipilimumab as first-line treatment for advanced nonsmall-cell lung cancer (CheckMate 012): results of an open-label, phase 1, multicohort study. Lancet Oncol 2017;18:31-41.

12. Antonia S, Goldberg SB, Balmanoukian A, et al. Safety and antitumour activity of durvalumab plus tremelimumab in non-small cell lung cancer: a multicentre, phase $1 \mathrm{~b}$ study. Lancet Oncol 2016;17:299-308.

13. AstraZeneca. AstraZeneca reports initial results from ongoing MYSTIC trial in stage IV lung cancer. Available online: https://www.astrazeneca.com/media-centre/pressreleases/2017/astrazeneca-reports-initial-results-from-the- ongoing-mystic-trial-in-stage-iv-lung-cancer-27072017. html. Accessed 17 October 2017.

14. United States Food and Drug Administration. FDA grants regular approval for pembrolizumab in combination with chemotherapy for first-line treatment of metastatic nonsquamous NSCLC. Available online: https://www.fda. gov/drugs/resources-information-approved-drugs/fdagrants-regular-approval-pembrolizumab-combinationchemotherapy-first-line-treatment-metastatic

15. United States Food and Drug Administration. FDA approves pembrolizumab in combination with chemotherapy for first-line treatment of metastatic squamous NSCLC. Available online: https://www.fda. gov/drugs/fda-approves-pembrolizumab-combinationchemotherapy-first-line-treatment-metastatic-squamousnsclc

16. United States Food and Drug Administration. FDA approves atezolizumab with chemotherapy and bevacizumab for first-line treatment of metastatic nonsquamous NSCLC. Available online: https://www.fda. gov/drugs/fda-approves-atezolizumab-chemotherapyand-bevacizumab-first-line-treatment-metastatic-nonsquamous. Accessed 25 July 2019.

17. Hellmann MD, Ciuleanu TE, Pluzanski A, et al. Nivolumab plus Ipilimumab in Lung Cancer with a High Tumor Mutational Burden. N Engl J Med 2018;378:2093-104.

18. Ready N, Hellmann MD, Awad MM, et al. First-Line Nivolumab Plus Ipilimumab in Advanced Non-SmallCell Lung Cancer (CheckMate 568): Outcomes by Programmed Death Ligand 1 and Tumor Mutational Burden as Biomarkers. J Clin Oncol 2019;37:992-1000.

19. Phull H. Biomarker or Bust: The TMB Journey in Lung Cancer. 2019. Available online: https:// www.medpagetoday.com/reading-room/asco/lungcancer/79743. Accessed 25 July 2019.
Cite this article as: Cummings AL, Santoso KM, Goldman JW. KEYNOTE-021 cohorts D and H suggest modest benefit in combining ipilimumab with pembrolizumab in secondline or later advanced non-small cell lung cancer treatment. Transl Lung Cancer Res 2019;8(5):706-709. doi: 10.21037/ tlcr.2019.08.11 